\title{
A Beam from Protvino to the Mediterranean Sea
}

\author{
V. Garkusha, S. Ivanov, A. Maksimov, F. Novoskoltsev, Y. Pimbursky, R. Sinyukov, \\ A.Sokolov, A. Zaitsev*
}

NRC "Kurchatov Institute" - Institute for High Energy Physics, Protvino, Moscow Region, 142281, Russia

\begin{abstract}
The report overviews preliminary results on the feasibility to produce a neutrino beam based on the U-70 proton synchrotron at Protvino for a very long baseline experiment with the deep water ORCA detector in the Mediterranean Sea.
\end{abstract}

\section{Generalities}

Juergen Brunner drew our attention $[1,2]$ to the fact that the U-70 accelerator [3] has the optimal energy ( $\approx 5 \mathrm{GeV}$ neutrino beam) and is located at the optimal distance $(2590 \mathrm{~km})$ from the ORCA facility [4] to provide a neutrino beam for experiments on mass hierarchy and $\mathrm{CP}$-violation. Here we report on the preliminary results of the feasibility study of producing a neutrino beam for such an experiment.

The layout of the entire Accelerator Complex U70 is shown in Fig. 1 [3]. It comprises four machines - 2 linear (I100, URAL30) and 2 circular (U1.5, U70) accelerators. The proton mode employs a cascade of URAL30 - U1.5 - U70, the light-ion (carbon) mode that of I100 - U1.5 - U70. The basic parameters of the three accelerators are presented in Table 1.

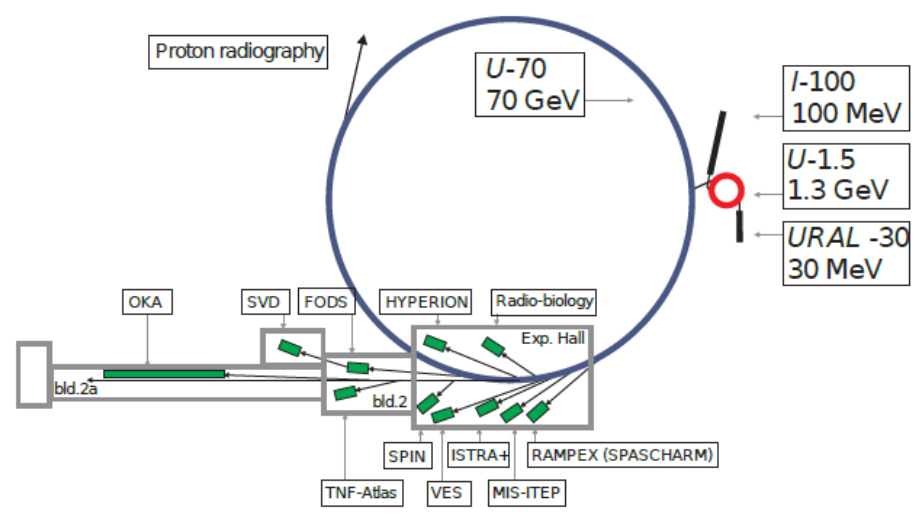

Figure 1. Accelerator Complex U70, beam transfer line network and fixed-target experimental facilities included.

*e-mail: alexandre.zaitsev@ihep.ru 
Table 1 Specification

\begin{tabular}{|lrl|lccl|}
\hline RFQ DTL & URAL30 & Synchrotrons & U1.5 & U70 & \\
\hline Energy, $E$ (protons) & $0.1-30$ & $\mathrm{MeV}$ & Energy, $E$ (protons) & $0.03-1.32$ & $1.32-69$ & $\mathrm{GeV}$ \\
Length, $L$ & 25.3 & $\mathrm{~m}$ & Orbit length, $L$ & 99.16 & 1483.699 & $\mathrm{~m}$ \\
Radiofrequency, $f_{\mathrm{RF}}$ & 148.5 & $\mathrm{MHz}$ & Curvature, $\rho$ & 5.73 & 194.125 & $\mathrm{~m}$ \\
Pulsed current, $I$ & 70 & $\mathrm{~mA}$ & Rigidity, $B \rho$ & $0.8-6.9$ & $6.87-233$ & $\mathrm{~T} \cdot \mathrm{m}$ \\
Pulse length, $t_{\mathrm{PT}}$ & $1-10$ & $\mu \mathrm{s}$ & Radio frequency & $0.8-2.8$ & $5.5-6.1$ & $\mathrm{MHz}$ \\
Cycle period, $T$ & 0.060 & $\mathrm{~s}$ & Intensity, $N$ & $2-9 \cdot 10^{11}$ & $1.5 \cdot 10^{13}$ & $\mathrm{ppp}$ \\
Sectioning & 5 & & Ramping time, $t_{\mathrm{R}}$ & 0.030 & 2.75 & $\mathrm{~s}$ \\
& & & Cycle period, $T$ & 0.060 & 9.77 & $\mathrm{~s}$ \\
& & & RF harmonic, $h$ & 1 & 30 & \\
\hline
\end{tabular}

In recent years, commonly the operational energy was about $50 \mathrm{GeV}$. This is a compromised value, which is still acceptable to users but noticeably minimizes electrical power consumption. The intensity $\sim 1 \cdot 10^{13} \mathrm{ppp}$ is limited mainly by the injector parameters. During the last few years, the experimental program was focused on a number of topics, in which the U-70 beam parameters allow to obtain valuable world class results such as:

- $\quad$ charged-kaon rare decays;

- $\quad$ hadron spectroscopy;

- $\quad$ physics of spin effects;

- hadron-nuclei interactions.

On top of that, the accelerator facility is used for applied research including proton radiography of fast processes and radiobiology with carbon nuclei.

Some time ago, neutrino experiments were an essential part of the scientific program at the U-70 machine $[5,6,7]$. Among the promising projects considered were also neutrino experiments with a very long baseline and a large deep-water detector [8]. At present, the neutrino program is frozen due to the limited intensity of the proton beam and the shortened running time ( 2 months per year) which does not leave chances for reaching competitive results in this field.

\section{Beam power}

The currently available beam power of about $15 \mathrm{~kW}$ might be increased in two stages. At the first stage, the beam power can reach $80 \mathrm{~kW}$ thanks to the use of multi-turn charge exchange $(\mathrm{H}-\rightarrow \mathrm{p})$ injection [9]. The second stage [10] requires the construction of a new injection complex optimized for providing a high intensity proton beam. The beam power in this case will reach $\approx 500 \mathrm{~kW}$.

At the first stage, the beam intensity is limited mainly by Coulomb tune shift [9] at the energy of injection from RFQ DTL URAL30 to the rapid cycling synchrotron U1.5. This intensity will be as high as $1.8 \cdot 10^{12} \mathrm{ppb}$ (compared with the current maximum intensity in a bunch $\mathrm{I}_{\max }=0.9 \cdot 10^{12} \mathrm{ppb}$ ). As a result, the maximal intensity of the accelerated beam in U70 will reach $5.2 \cdot 10^{13} \mathrm{ppp}$.

The acceleration cycle consists of the following steps:

- injection from URAL30 to U1.5,

$\mathrm{t}=2.2 \mathrm{~s}$

- acceleration to $69 \mathrm{GeV}$,

$\mathrm{t}=2.4 \mathrm{~s}$

- beam extraction,

$\mathrm{t}=0.2 \mathrm{~s}$

- magnetic field return to injection flat-bottom,

$\mathrm{t}=2.4 \mathrm{~s}$

- cycle in total,

$\mathrm{T}=7.2 \mathrm{~s}$ 
Further shortening of the cycle requires a significant upgrade of the power supply and accelerating systems. It is not planned for the first stage. The accelerating stations of U1.5 and $\mathrm{U} 70$ can be adapted to new beam parameters within a moderate upgrade.

As a result, the maximal beam power will reach $80 \mathrm{~kW}$. Increasing the beam power is among the top priorities of the laboratory. The work for the intensity increasing includes an extensive list of activities:

- Construction of a source of H-minus ions and charge-exchange-injection to U1.5

- Re-alignment of the lattice for 120 of $200 \mathrm{t}$ magnets to reach sub-mm accuracy

- Electrical smoothing of the vacuum chamber

- Enhancing the B-field correction, especially the orbit one

- Longitudinal beam feedback, $3 \mathrm{MHz}$ bandwidth (two transverse ones are operational)

- Better handling of gamma-transition crossing

- Optimization of equipment layout to overcome the deficit of free space along orbit

- Upgrade of the water cooling system

- Possibly also a new system for a high-intensity beam abort.

A further increase in the intensity of the beam, up to $\approx 500 \mathrm{~kW}$, requires the construction of a new injection complex (Fig. 2). This large-scale project, proposed several years ago [10], will open new opportunities for experiments in high-energy physics and will allow solving a wide range of applied tasks.

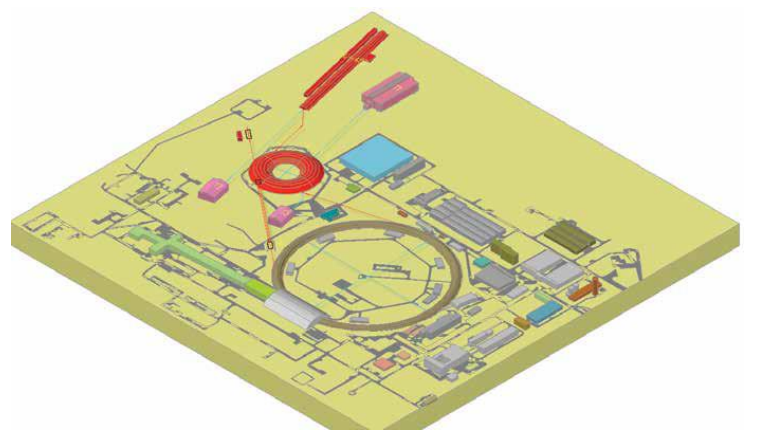

Figure 2 (from ref. [10]). Location of the Facility for Intense Hadron Beams:

1 - Linear accelerator LU-400 (red color);

2 - Rapid cycling synchrotron U-3.5 (red color);

3 - Existing building (blue color) will be used for the technical support. Light-gray and yellow colors mark the existing infrastructure. In the center - the U-70 ring.

At present, this project is suspended. A number of systems are under conceptual development.

\section{Neutrino channel}

The existing single-turn beam extraction system enables the proton beam transport to the southern part of the main experimental hall (Fig. 3). To be directed to ORCA the proton beam has to be bent by $-11.8^{\circ}$ in the vertical and $\approx 20^{\circ}$ in the horizontal plane. The 
magnetic optics, which provides beam turns and transportation to the target station, will be located partly in the experimental hall, and partly outside.
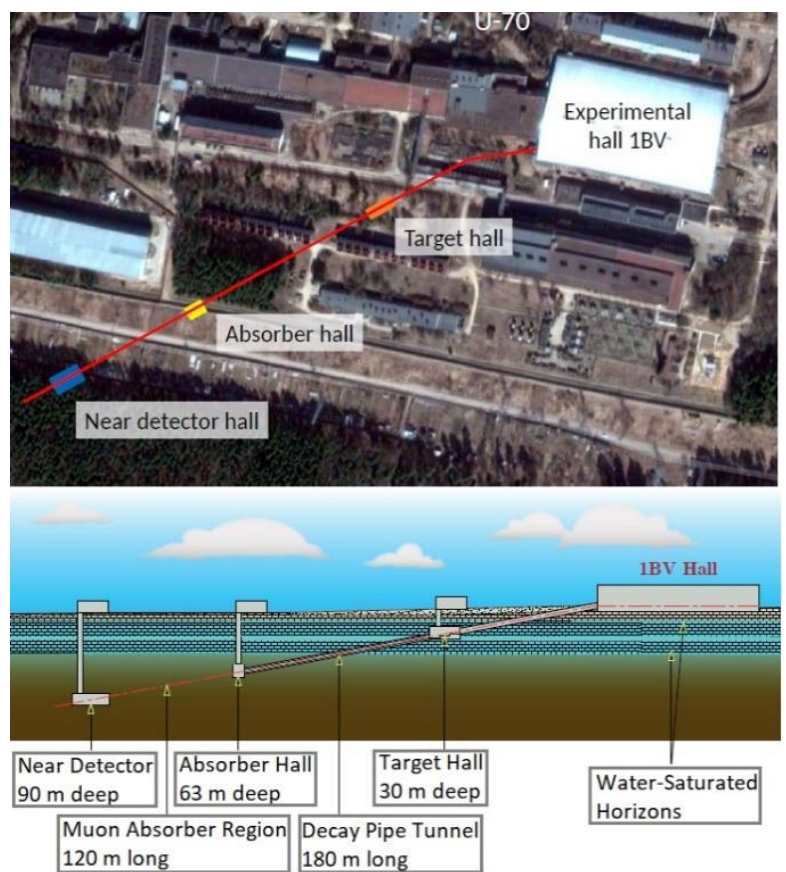

Figure 3. Top view and elevation view of the neutrino channel.

The target hall should be located at a depth of $30 \mathrm{~m}$. An earlier geological survey in the planned area of construction of the neutrino channel revealed two water-saturated horizons located at depths of 15 and 35 meters. The construction of a target hall with a high radiation load just near the water-saturated horizon may require considerable effort and cost to ensure the baseline environmental safety. In this hall there will be a target ( $1 \mathrm{~m} \mathrm{long}, 5$ $\mathrm{mm}$ diameter graphite rod) with a cooling system, two horns focusing system (Fig.4), radiation shielding, crane, tools for remote manipulation of irradiated equipment, beam monitors, radiation monitors, vacuum equipment, ventilation. The hall dimensions are preliminary estimated as $9 \times 12 \times 24 \mathrm{~m}^{3}(\mathrm{~W} \times \mathrm{H} \times \mathrm{L})$.

The decay channel could have a length of $180 \mathrm{~m}$ and a diameter of $2 \mathrm{~m}$. The vacuum volume will be equipped with a water-cooling system and will be surrounded by concrete shielding.
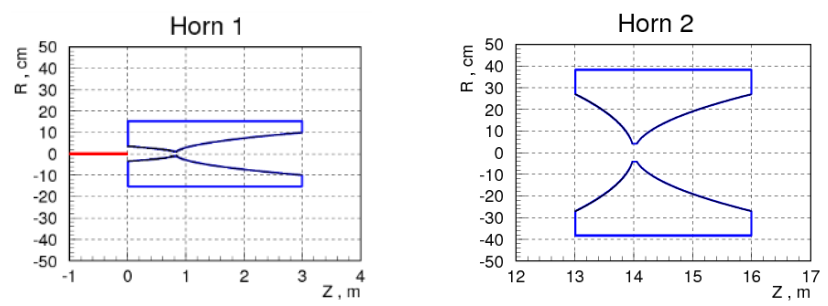

Figure 4. Two horns scheme of the focusing system. Horn currents are equal to $220 \mathrm{kA}$. 
The absorber hall should be located at a depth of $63 \mathrm{~m}$. It houses the hadron absorber, monitors of muon fluxes, radiation monitors, elements of the vacuum system, devices for loading massive hadron absorber and ventilation. The hall dimensions are estimated as $6 \times 12 \times 12 \mathrm{~m}^{3}$.

The near detector hall might be located at a distance of $120 \mathrm{~m}$ from the absorber hall, at a depth of $90 \mathrm{~m}$. This distance provides reliable muon absorption. This hall houses the near neutrino detector for monitoring neutrino fluxes. The head of the hall is free from equipment. It is needed to reduce the flux of muons produced by neutrino interactions in the soil and reaching the detector. The hall dimensions are estimated as $9 \times 12 \times 36 \mathrm{~m}^{3}$. The mass of the detector is bounded from above by the number of neutrino interactions in one bunch to avoid accidental coincidences, and from below by the desired statistics. A reasonable compromise is achieved with a mass of several tons. In addition, it is desirable to have water in the detector to avoid recalculation of the cross sections from one nuclear target to another, good identification of reaction channels and good energy resolution. The last requirement arises because the neutrino spectra in the near and far detectors differ significantly (Fig. 5). Accurate measurement of the energy at the near detector will help to reduce the systematic errors caused by this difference.

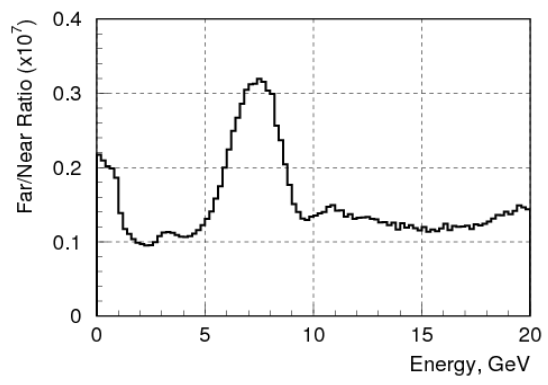

Figure 5. The ratio of the neutrino flux per square meter at the far detector (ORCA) to the flux per square meter at the near detector with an area of $2 \times 2 \mathrm{~m}^{2}$.

Figure 6 shows the calculated neutrino spectra at the ORCA site. They are normalized to $4 \cdot 10^{20}$ protons on the target, which corresponds to 5 years run with a beam of $90 \mathrm{~kW}(30$ month of running with efficiency $\varepsilon \approx 60 \%$ ). The focusing system is optimized for the maximum of the neutrino flux at energy $\mathrm{E} \approx 5 \mathrm{GeV}$.
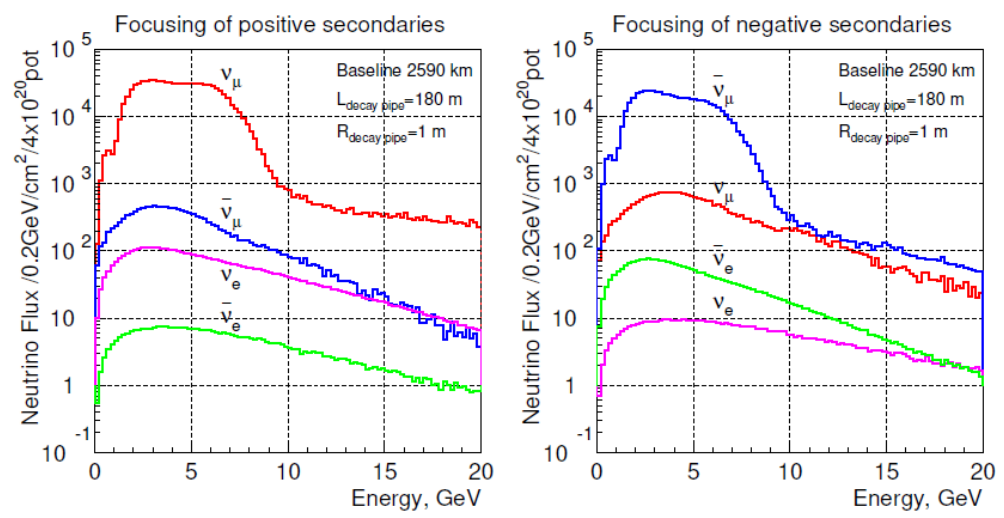

Figure 6. Neutrino (left) and antineutrino (right) spectra at the ORCA site. 
Figure 7 shows the distribution over the distance of neutrino interaction point from the beam center in the plane perpendicular to the beam in the region of the ORCA detector. This distribution is useful for estimating the required accuracy of beam pointing at the detector and the corresponding systematic errors. Preliminary estimates show that in order to achieve accuracy in neutrino fluxes at a level of a percent, the accuracy of aiming should be $\approx 0.6 \mathrm{mrad}$

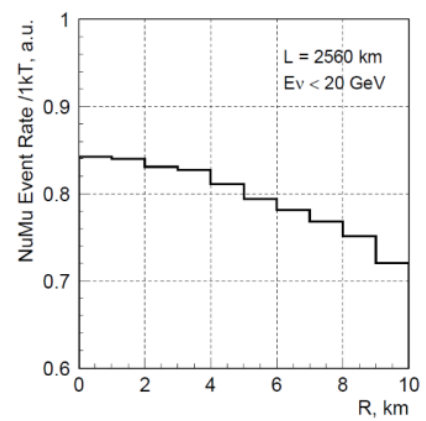

Figure 7. Distribution of neutrino interactions perpendicular to the beam at the ORCA site.

\section{Conclusions}

The neutrino beam from the U-70 accelerator (with an increased intensity) towards ORCA has a good potential for study neutrino oscillations at a new level. At this stage of the analysis, no stopping factors of technical nature are found to increase the power of the U-70 beam to $80 \mathrm{~kW}$.

The construction of a new injection complex (OMEGA) will put forward unique opportunities for neutrino experiments.

The concept of long base neutrino experiments in hand - "a high-intensity neutrino beam to a very large volume deep-water detector" - deserves further detailed study.

\section{References}

1. J. Brunner, Advances in High Energy Physics, Volume 2013, p. 1-16, (2013), http://dx.doi.org/10.1155/2013/782538.

2. J. Brunner, Proc. of Neutrino 2018, Heidelberg, 4-9 June, (2018) https://doi.org/10.5281/zenodo.1300743

3. S. Ivanov, Proc. of RUPAC-2016, St. Petersburg, p. 44-47, (2016)

4. KM3NeT Collaboration: S. Adrian-Martinez et al., J. Phys. G: Nucl. Part. Phys. 43, №8, $130 \mathrm{pp},(2016)$.

5. A.P. Bougorsky et al., Yad. Fiz. 28, 424 (1978).

6. V.V. Ammosov et al., Phys. Elem. Part. Atom. Nucl. 23, 648 (1992).

7. L.S. Barabash et al., Preprint JINR P13-2002-152, 52 pp, (2002), http://cds.cern.ch/record/748253 (in Russian).

8. A.S. Arefev et al., Preprint IHEP-94-36, 24 pp, (1994) (In Russian).

9. V. I. Balbekov et al. (ed.), Preprint IHEP-93-27, 230 pp, (1993) (in Russian).

10. N. E. Tyurin et al., News and Problems of Fundamental Physics 2 (9) 2010, IHEP,

Protvino , 54 pp, (2010), http://ihep.ru/files/IHEP-2-10.pdf 